\title{
CALCULATING DEFORMATION TEXTURES FOR CUBIC POLYCRYSTALS
}

\author{
V. D. SOLOVEI and V. L. KOLMOGOROV \\ Institute of the Engineering Science, 91 Pervomaiskaja Str., \\ 620219 Ekaterinburg GSP-207, Russia
}

(Received 8 September 1994)

The analytical dependences for the components of the additional rotation vector of the grain lattice* in a cubic polycrystal on the strain components describing approximately analogous dependences, determined by the Bishop and Hill deformation texture theory (Bishop and Hill, 1951), are proposed.

KEY WORDS: Deformation textures, Taylor-Bishop-Hill model, analytical approximation, cubic crystals.

\section{NOTATION}

$\omega_{i}$-grain lattice rotation vector in the polycrystal

$u_{i}$-displacement vector of a macroscopic particle consisting of a great number of grains

$\varepsilon_{i j k}-$ Levi-Civita tensor

$\omega_{i}^{a}$-additional rotation vector of the grain lattice, connected with the acting crystallographic slip systems

$\varepsilon_{i j}$-plastic strain tensor

$\gamma_{\alpha}$-plastic deformation components: ${ }^{* *} \gamma_{1}=\varepsilon_{11}, \gamma_{2}=\varepsilon_{22}, \gamma_{3}=\varepsilon_{33}, \gamma_{4}=2 \varepsilon_{23}, \gamma_{5}=2 \varepsilon_{31}$, $\gamma_{6}=2 \varepsilon_{12}$

$a_{i}^{(n)}$-single vector of the shear direction for the n-th slip system

$b_{i}^{(n)}$-single vector of the normal to the slip plane for the $n$-th slip system

$\delta_{n}$-shear on the $n$-th slip system

"Grain lattice means crystalline grain lattice.

"*The values of the Latin indices 1,2,3 are referred to the principal axes of the cubic crystal $x_{1}$, $x_{2}, x_{3}$ respectively. 


\section{INTRODUCTION}

The lattice rotation vector of the arbitrary grain in the deformed polycrystal is represented by formula (Taylor, 1938; Bunge, 1970; Houtte and Aernoudt, 1975)

$$
\omega_{i}=1 / 2 \varepsilon_{i j k} u_{k, j}+\omega_{i}^{a} .
$$

The displacement vector curl $1 / 2 \varepsilon_{i j k} u_{k, j}$ represents a common (rigid) rotation of a polycrystal macroscopic particle and, in case of inhomogeneous deformation, it can be found from the boundary value problem of the continua mechanics.

The vector of the additional rotation of the grain lattice $\omega_{i}^{a}$ is connected with the acting slip systems $\{111\}<110>$ and $\{110\}<111>,\{112\}<111>,\{123\}<111>$ for the FCC and BCC crystals respectively.

In the theories using the Taylor approximation (Taylor, 1938) it is assumed that the strain of the arbitrary grain is equal to the macroscopic strain.

Virtual sets of five slip systems and the shears on them, according to Taylor's deformation texture theory (Taylor, 1938), must provide the given strain

$$
\varepsilon_{i j}=1 / 2 \sum_{n=1}^{5}\left(a_{i}^{(n)} b_{j}^{(n)}+a_{j}^{(n)} b_{i}^{(n)}\right) \delta_{n},
$$

where summation spreads over the all slip systems of the virtual set of the slip systems.

According to the Bishop and Hill deformation texture theory (Bishop and Hill, 1951), the virtual sets of five slip systems and the shears on them must also satisfy the principle of the maximum strain work for the grain. The last requirement decreases the number of the virtual sets of five slip systems.

The acting sets of five slip systems and the shears on them are found among the virtual ones by means of the minimum criterion of the shears moduli sum on the slip systems (Taylor, 1938), which can be written as

$$
\sum_{n=1}^{5}\left|\delta_{n}\right| \rightarrow \min
$$

Several acting sets of five slip systems can exist in general.

The vector of the additional rotation of the grain lattice is determined by the shears on the acting slip systems and can be written in the following form:

$$
\omega_{i}^{a}=-1 / 2 \varepsilon_{i j k} \sum_{n=1}^{5}\left(b_{j}^{(n)} a_{k}^{(n)}-b_{k}^{(n)} a_{j}^{(n)}\right) \delta_{n} .
$$

If several acting sets of five slip systems exist, the additional rotation of the grain lattice is found as the mean rotation for all these sets.

The dependences for the components of the additional rotation vector of the grain lattice on the strain components is determined by the relations (2)-(4)* implicitly. In the present paper explicit analytical dependences for the components of the additional rotation vector of cubic grain lattice on the strain components are proposed.

${ }^{*}$ The principle of maximum strain work for the crystal must also be satisfied in the case of the Bishop and Hill deformation texture theory. 


\section{FINDING THE UNKNOWN ANALYTICAL DEPENDENCES}

The dependences for the components of the additional rotation vector of the grain lattice on the strain components, determined by relations (2)-(4), are the homogeneous functions of the first degree of homogeneity and can be represented by means of the following functions*

$$
\omega_{i}^{a}=\sqrt{\gamma_{\beta} \gamma_{\beta}} f_{i}\left(n_{\alpha}\right)
$$

where $n_{\alpha}=\gamma_{\alpha} / \sqrt{\gamma_{\beta} \gamma_{\beta}}$.

The multinomials $f_{i}\left(n_{\alpha}\right)$ are odd functions and must consist only of the odd degrees of the arguments. We take into account only the terms having the first and the third degrees of the arguments

$$
f_{i}\left(n_{\alpha}\right)=a_{\alpha}^{(i)} n_{\alpha}+a_{\alpha \beta \gamma}^{(i)} n_{\alpha} n_{\beta} n_{\gamma} .
$$

Let us consider the effect of the symmetry elements of the cubic crystalline lattice on the coefficients of the multinomials (6).

First we consider the function $f_{3}\left(n_{\alpha}\right)$. The coefficients of the multinomial $f_{3}\left(n_{\alpha}\right)$, having the even number of the indices 5 and 6 or 4 and 6 , are equal to zero. This results from the symmetry axes of the second order $O x_{1}$ or $O x_{2}$ respectively. The coefficients of this multinomial, having the odd number of indices 4 and 5 , equal zero since the axis of the second order $O x_{3}$ exists. The symmetry axis of the fourth order $O x_{3}$ causes the fact that the rezidual non-zero coefficients of the multinomial $f_{3}\left(n_{\alpha}\right)$ satisfy the following relations:

$$
\begin{aligned}
& a_{226}^{(3)}=-a_{116}^{(3)}, \\
& a_{556}^{(3)}=-a_{446}^{(3)}, \\
& a_{236}^{(3)}=-a_{136}^{(3)}, \\
& a_{245}^{(3)}=-a_{145}^{(3)} .
\end{aligned}
$$

The symmetry axes of the fourth order $O x_{1}$ and $O x_{2}$ do not give new results.

We choose new variables $\gamma_{1}, \gamma_{2}, \gamma_{4}, \gamma_{5}, \gamma_{6}$ taking into account the incompressibility condition of plastic strain of a crystal. Then the variables $n_{1}, n_{2}, n_{4}, n_{5}, n_{6}$ will be the new variables for the multinomials (6) ${ }^{* *}$. The non-zero coefficients of the multinomial $f_{3}\left(n_{\alpha}\right)$, transformed into the new variables, satisfy the following relations:

$$
\begin{aligned}
& a_{226}^{(3)}=-a_{116}^{(3)}, \\
& a_{556}^{(3)}=-a_{446}^{(3)}, \\
& a_{245}^{(3)}=-a_{145}^{(3)} .
\end{aligned}
$$

On the one hand, the relations for the coefficients of the multinomials $f_{1}\left(n_{\alpha}\right)$ and $f_{2}\left(n_{\alpha}\right)$, analogous to the relations (7), can be found by using the above-mentioned symmetry considerations. On the other hand, these coefficients can be found by means of the transformations of the multinomial $f_{3}\left(n_{\alpha}\right)$ connected with the circular permutations

${ }^{*}$ For the recurrent Greek indices, summation on all their possible values 1, .., 6 is assumed.

** For the new variables there is the equality $n_{1}+n_{2}+n_{3}=0$ 
of the coordinates $x_{1} \rightarrow x_{2}, x_{2} \rightarrow x_{3}, x_{3} \rightarrow x_{1}$. Comparing the corresponding coefficients found by these two methods and taking into account relations (7), we express the coefficients of the multinomials $f_{1}\left(n_{\alpha}\right)$ and $f_{2}\left(n_{\alpha}\right)$ through the coefficients of the multinomial $f_{3}\left(n_{\alpha}\right)$ by the following relations:

$$
\begin{array}{ll}
a_{114}^{(1)}=-a_{116}^{(3)}, & a_{225}^{(2)}=a_{116}^{(3)}, \\
a_{124}^{(1)}=-2 a_{116}^{(3)}, & a_{125}^{(2)}=2 a_{116}^{(3)}, \\
a_{554}^{(1)}=a_{446}^{(3)}, & a_{445}^{(2)}=-a_{446}^{(3)}, \\
a_{664}^{(1)}=-a_{446}^{(3)}, & a_{665}^{(2)}=a_{446}^{(3)}, \\
a_{156}^{(1)}=a_{145}^{(3)}, & a_{146}^{(2)}=-2 a_{145}^{(3)}, \\
a_{256}^{(1)}=2 a_{145}^{(3)}, & a_{246}^{(2)}=-a_{145}^{(3)} .
\end{array}
$$

From (7) and (8) it is seen that the non-zero coefficients of the multinomials (6) are expressed through three independent coefficients $a_{116}^{(3)}, a_{446}^{(3)}$ and $a_{145}^{(3)}$.

The values of the independent coefficients $a_{116}^{(3)}, a_{446}^{(3)}$ and $a_{145}^{(3)}$ are calculated in the following way. As the functions (5) are odd functions, in the space of the new variables $\gamma_{1}, \gamma_{2}, \gamma_{4}, \gamma_{5}, \gamma_{6}$ with the intervals $\gamma_{\alpha}=0.0, \ldots, 0.25$ (steps $\Delta \gamma_{\alpha}=0.05$ ), for all $\gamma_{\alpha}$, we build a net consisting of $6^{5}$ equidistant nodes. Then the components of the additional rotation vector for FCC crystals are calculated numerically in the net nodes (except the nodes in which the equalities $\gamma_{4}=\gamma_{5}=\gamma_{6}=0$ take place) according to Bishop and Hill deformation texture theory (Bishop and Hill, 1951). We find the unknown independent coefficient approximating (by the method of least squares) the abovementioned components of the additional rotation vector for FCC crystals calculated in the net nodes by the functions (5). The calculations give:

$$
\begin{aligned}
& a_{116}^{(3)}=-1.51, \\
& a_{446}^{(3)}=-0.89, \\
& a_{145}^{(3)}=1.25 .
\end{aligned}
$$

The tension axes rotations of the FCC crystals elongated by the 5 per cent along the different crystallographic directions, calculated by the Bishop and Hill deformation texture theory, are shown in Figure 1. Analogous rotation field for the tension axes of the FCC crystal, calculated by means of the here found relations (5)-(8) and data (9), is shown in Figure 2. It is obvious that the rotation fields of the tension axes of the FCC crystals represented in Figures 1 and 2 are close.

\section{CONCLUSION}

The analytical expressions found here represent the dependence of the additional rotation of the grain lattice for FCC crystal on strain in the explicit form. They can be used to calculate approximately additional rotations of the grain lattices in accordance with the Bishop and Hill deformation texture theory for the FCC polycrystals and BCC polycrystals deformed by slipping only on the slip systems $\{110\}<111>$. In the latter case the signs of the independent coefficients (9) must be changed. Using the found analytical dependences one can essentially decrease computing time for the calculation of the additional rotations of the grain lattices occurring during the deformation of polycrystals. 


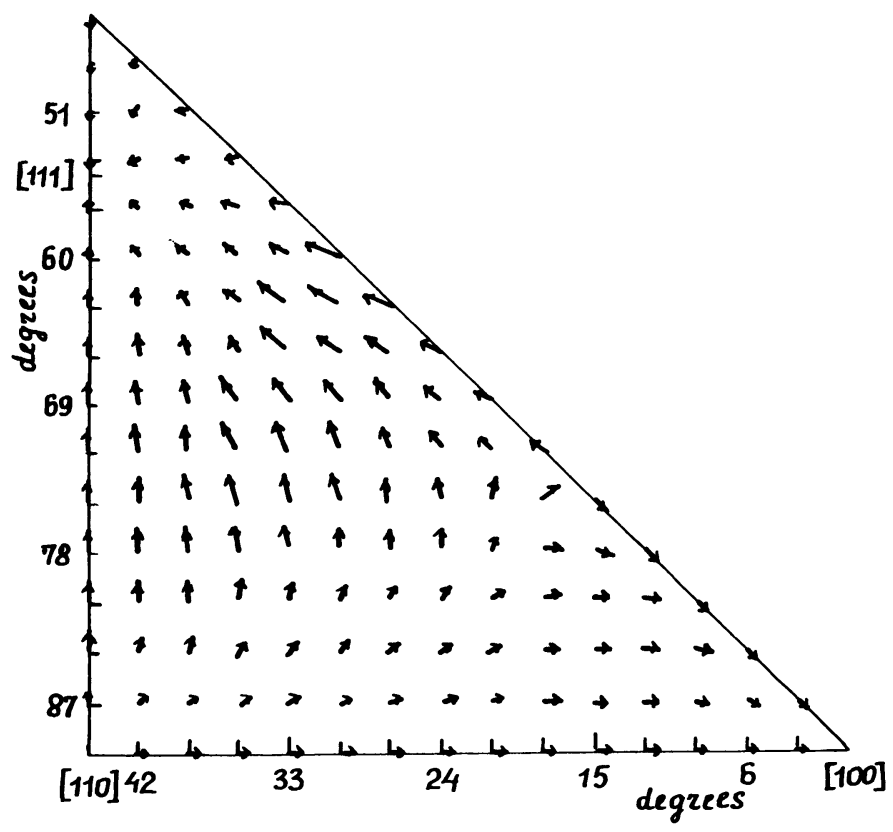

Figure 1 Rotations of the tensile axes of the FCC crystals, found by means of Bishop and Hill theory.

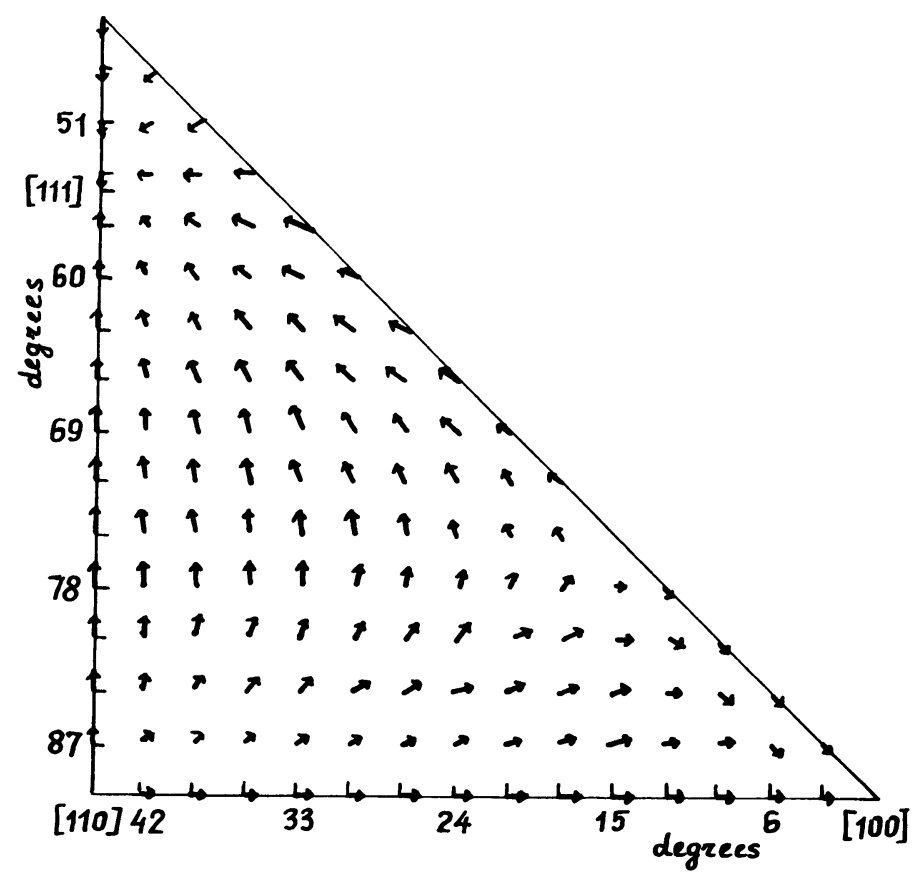

Figure 2 Rotations of the tensile axes of the FCC crystals, found by means of approximation functions. 


\section{References}

Bishop, J. F. W. and Hill, R. (1951). A Theoretical Derivation of the Plastic Properties of a Polycrystalline Face-Centred Metal. Philosophical Magazine, 42, 1298-1307.

Bunge, H. J. (1970). Some Application of the Taylor Theory of Polycrystal Plasticity. Kristall und Technik, 5, 145-175.

Houtte, P. and Aernoudt, E. (1975). Solution of the Generalized Taylor Theory of Plastic Flow. Part 2. Zeitschrift fur Metallkunde, 66, 202-209.

Taylor, G. I. (1938). Plastic strain in metals. Journal of the Institute of Metals, 62, 307-324. 